\title{
P58 Structured Exercise Training is Associated with Better Post Occlusive Reactive Hyperaemia in Skeletal Muscle Measured using Near Infrared Spectroscopy (NIRS)
}

\author{
Chirag Rao ${ }^{1, *}$, Siana Jones ${ }^{1}$, Khadija Ezzi ${ }^{1}$, Alun Hughes ${ }^{1}$ \\ ${ }^{1}$ UCL Institute of Cardiovascular Science, London, United Kingdom
}

\section{ABSTRACT}

Background: Participation in structured exercise training may improve microvascular function. Post occlusive reactive hyperaemia (PORH) is a measure of microvascular function in skeletal muscle and can be assessed using near-infrared spectroscopy (NIRS). We examined PORH following a short and a long arterial occlusion to test whether individuals who participate in more structured exercise have better PORH.

Methods: 18 healthy volunteers (mean age $=23.1$ (standard deviation $(\mathrm{SD})=4.9 \mathrm{y}$ ), male $=12)$ underwent $30 \mathrm{~s}$ and 5 minute arterial occlusions of the left lower leg using a cuff inflated to supra-systolic pressure. Change in oxygenated haemoglobin concentration (oxy- $\mathrm{Hb})$ was measured from the gastrocnemius using NIRS (Artinis, Netherlands). PORH was measured as time to $50 \%\left(\mathrm{~T}_{50}\right)$ of peak oxy-Hb following cuff release and a lower $\mathrm{T}_{50}$ indicates a better response. Exercise participation was categorised into high $(n=12)$ or low $(n=6)$ based on self-reported hours of structured exercise per week (high $\geq 4 \mathrm{hr}$ per week). Results are given as median (IQR) and statistical comparison was by the Mann Whitney U-Test.

Results: High exercise participants had a greater PORH response than low exercise participants $\left(\mathrm{T}_{50}: 8.0(6.2,9.5) \mathrm{s}\right.$ vs 12.5 $(8.6,27.2) \mathrm{s}, p=0.04$; Figure 1a). The hyperaemic response to $30 \mathrm{~s}$ occlusion was also better in the high exercise group $\left(\mathrm{T}_{50}\right.$ : $2.4(1.7,6.6) \mathrm{s}$ vs $4.3(3.6,4.9) \mathrm{s} p=0.17$; Figure $1 \mathrm{~b})$, although this difference was not statistically significant.

Conclusion: Individuals who participate in more structured exercise have evidence of improved microvascular function in skeletal muscle.

a

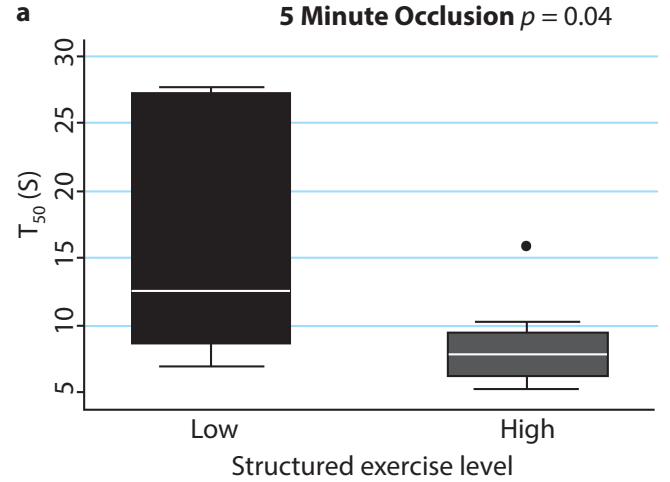

b

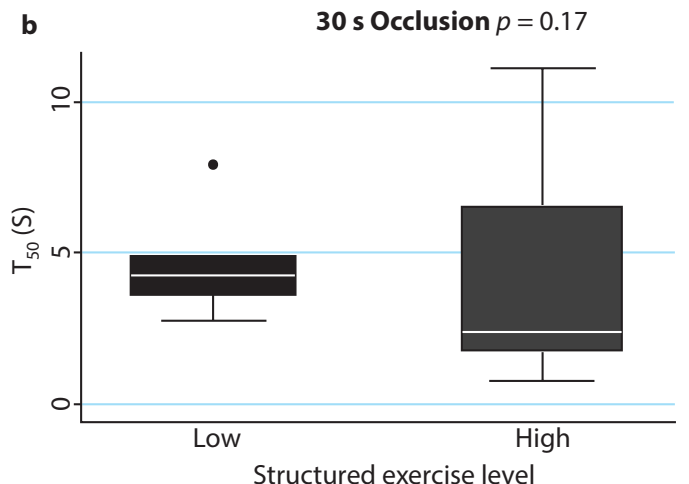

Figure $1 \mid$ a \& b Boxplots comparing the PORH response in low and high exercise groups. Data are $12.5(8.6,27.2) s$ and $8.0(6.2,9.5) \mathrm{s}$ for the low exercise group and the high exercise group respectively in the 5 minute occlusion compared with $4.3(3.6,4.9)$ s and $2.4(1.7,6.6) \mathrm{s}$ in the 30 s occlusion. $p$-values are calculated using a Mann Whitney $U$-Test.

(c) 2019 Association for Research into Arterial Structure and Physiology. Publishing services by Atlantis Press International B.V. This is an open access article distributed under the CC BY-NC 4.0 license (http://creativecommons.org/licenses/by-nc/4.0/). 